\title{
A BMI optimization approach to robust output-feedback control*
}

\author{
S. Kanev, C. Scherer, M. Verhaegen, and B. De Schutter
}

If you want to cite this report, please use the following reference instead: S. Kanev, C. Scherer, M. Verhaegen, and B. De Schutter, "A BMI optimization approach to robust output-feedback control," Proceedings of the 42nd IEEE Conference on Decision and Control, Maui, Hawaii, pp. 851-856, Dec. 2003.

Delft Center for Systems and Control

Delft University of Technology

Mekelweg 2, 2628 CD Delft

The Netherlands

phone: +31-15-278.24.73 (secretary)

URL: https: //www.dcsc.tudelft.nl

*This report can also be downloaded viahttps://pub.deschutter.info/abs/03_004.html 


\title{
A BMI Optimization Approach to Robust Output-Feedback Control
}

\author{
Stoyan Kanev, Carsten Scherer, Michel Verhaegen and Bart De Schutter \\ Delft Center for Systems and Control \\ Delft University of Technology \\ Mekelweg 2, 2628 CD DELFT, The Netherlands \\ \{s.kanev, m. verhaegen, b. deschutter, c.w.scherer\}@dcsc.tudelft.nl
}

\begin{abstract}
In this paper a new approach is proposed to design locally optimal robust output-feedback controllers. It is iterative by nature, and starting from any initial feasible controller it performs local optimization over a suitably defined non-convex function at each iteration. The approach features the properties of computational efficiency, guaranteed convergence to a local optimum, and applicability to a very wide range of problems. The paper also proposes a fast procedure for initially feasible controller computation based on LMIs. The design objectives considered are $\mathcal{H}_{2}, \mathcal{H}_{\infty}$, and pole-placement constraints. The procedure consists of two steps: first an optimal robust mixed $\mathcal{H}_{2} / \mathcal{H}_{\infty}$ /pole-placement state-feedback gain is designed, which is consequently kept fixed at the second step during the design of the remaining controller matrices. The approach is demonstrated on a model of one joint of a real-life space robotic manipulator.
\end{abstract}

\section{INTRODUCTION}

It is well-known that the problem of output-feedback controller design in the presence of polytopic model uncertainty is not convex and can be represented in terms of bilinear (or rather bi-affine) matrix inequalities (BMIs) [17]. These, however, are in general NPhard [15], which means that any algorithm which is guaranteed to find the global optimum cannot be expected to have a polynomial time complexity.

There exist different approaches to the solution of the BMI problem, which can be classified into global [3], [5], [16], [18] and local [8], [9], [7], [6]. Most of the global algorithms to the BMI problem are variations of the Branch and Bound Algorithm [16], [3]. Although the major focus of all global search algorithms is the computational complexity, none of them is polynomial-time due to the NP-hardness of the problem. As a result, these approaches can currently be applied only to problems of modest size with no more than just a few "complicating variables" [16]. Thus, the global algorithms are at present not practical to output-feedback controller design problems for polytopic systems, where even small problems can result in lots of such complicating variables (for instance, in the case study presented in Section $\S$ VI there are 40 complicating variables).

Most of the existing local approaches, on the other hand, are computationally fast but, depending on the initial condition, may not converge to the global optimum. The simplest local approach makes use of the fact that by fixing some of the variables, $\boldsymbol{x}$, the BMI problem becomes convex in the remaining variables $\boldsymbol{y}$, and vice versa, and iterates between them [9]. This is also the idea behind the well-known $D-K$ iteration for $\mu$-synthesis. In some papers [9], [10] the search is performed in other more suitably defined search directions. Nevertheless, these type of algorithms, called coordinate descent methods in [9], Alternating SDP Method in [3], and the dual iteration in [9], are not guaranteed to converge to a local solution [5], [3], [18].

*This work is sponsored by the Dutch Technology Foundation (STW) under project number DEL.4506.
Another local approach is the so-called Path-Following Method [7], which is based on linearization. The idea is that under the assumption of small search steps the BMI problem can be approximated as an LMI problem by making use of the first-order perturbation approximation [7]. In practise this approach can be used for problems where the required closed-loop performance is not drastically better than the open-loop system performance, to solve the actuator/sensor placement problem, as well as the controller topology design problem [7]. Yet another local approach is the Rank-Minimization Method [8]. Although convergence is established for a suitably modified problem, there are no guarantees that the solution to this modified problem will be feasible for the original BMI problem. The $X Y$-Centering Algorithm, proposed in [10] is also an alternative local approach, which focusses on a subclass of BMI problems in which the non-convexity can be expressed in the form $X=Y^{-1}$, and is thus applicable to a restricted class of controller design problems. Finally, the Method of Centers (MC) has guaranteed local convergence provided that a feasible initial condition is given [5]. It is, however, the computationally most involving approach, and it is also known that it can experience numerical problems at later iterations [3].

Similarly to the MC, the approach in this paper performs local optimization over a suitably defined non-convex function at each iteration. It enjoys the property of guaranteed convergence to a local optimum, while at the same time it is computationally faster and numerically more reliable than the MC. In addition to that, a twostep procedure is proposed for the design of an initially feasible controller. At the first step an optimal robust mixed $\mathcal{H}_{2} / \mathcal{H}_{\infty} /$ poleplacement state-feedback gain $F$ is designed. This gain $F$ is consequently kept fixed during the design of the remaining statespace matrices of the dynamic output-feedback controller. Although the first step is convex, the second one remains non-convex. However, by constraining a Lyapunov function for the closed-loop system to have a block-diagonal structure, this second step is easily transformed into an LMI optimization problem. Somewhat related is the work of [14], where the authors attack a specific BMI problem with quadratic terms by constructing LMIs. This is achieved at the expense of some conservatism that results from an over-bounding of the non-affine quadratic terms leading to the LMIs.

The paper is organized as follows. In Sec. II the notation is defined and the problem is formulated. The proposed algorithm for locally optimal controller design is next presented in Sec. III. For the purposes of its initialization, an approach to initially feasible controller computation is proposed in Sec. IV, where a multiobjective criterion is considered. A summary of the complete algorithm is given in Sec. V. In Sec. VI the design approach is tested on a model of one joint of a real-life space robotic manipulator. Finally, Sec. VII concludes the paper. 


\section{PROBLEM FORMULATION}

\section{A. Notation}

The symbol $\bullet$ in LMIs will denote entries that follow from symmetry. In addition to that the notation $\operatorname{Sym}(A)=A+A^{*}$ will also be used. Boldface capital letters denote variable matrices appearing in matrix inequalities, and boldface small letters - vector variables. The convex hull of a set of matrices $\mathcal{S}=\left\{M_{1}, \ldots, M_{N}\right\}$ is denoted as $\operatorname{co}\{\mathcal{S}\}$. Also used is the notation $\langle A, B\rangle=\operatorname{trace}\left(A^{T} B\right)$. The set of eigenvalues of a matrix $A$ will be denoted as $\lambda(A)$. The symbol $\triangleq$ will denote "equal by definition". The direct sum of matrices $A_{i}$, $i=1,2, \ldots, n$ will be denoted as $\bigoplus_{i=1}^{n} A_{i}=A_{1} \oplus \cdots \oplus A_{n} \triangleq$ blockdiag $\left(A_{1}, \ldots, A_{n}\right)$. Also, $v_{i}$ will denote the $i$-th element of the vector $v$. The projection onto the cone of symmetric positive-definite matrices is defined as $[A]^{+}=\arg \min _{\boldsymbol{S} \geq 0}\|A-\boldsymbol{S}\|_{F}$. Similarly, the projection onto the cone of symmetric negative-definite matrices is defined as $[A]^{-}=\arg \min _{\boldsymbol{S} \leq 0}\|A-\boldsymbol{S}\|_{F}$. Some useful properties of these projections can be found in [1]. Finally, $A \otimes B$ denotes the Kronecker product of $A$ and $B$.

\section{B. Problem Formulation}

Consider the following uncertain system

$$
\mathcal{S}_{\sigma}:\left\{\begin{aligned}
\sigma x & =A^{\Delta} x+B_{\xi}^{\Delta} \xi+B_{u}^{\Delta} u \\
z & =C_{z}^{\Delta} x+D_{z \xi}^{\Delta} \xi+D_{z u}^{\Delta} u \\
y & =C_{y}^{\Delta} x+D_{y \xi}^{\Delta} \xi+D_{y u}^{\Delta} u
\end{aligned}\right.
$$

where $x(t) \in \mathbb{R}^{n}$ is the system state, $z(t) \in \mathbb{R}^{n_{z}}$ is the controlled output of the system, $u(t) \in \mathbb{R}^{m}$ is the control action, and $y(t) \in$ $\mathbb{R}^{p}$ is the measured output and $\xi(t) \in \mathbb{R}^{n_{\xi}}$ is the disturbance to the system, and where the symbol $\sigma$ represents the $s$-operator (i.e. the time-derivative operator) for continuous-time systems, and the $z$-operator (i.e. the shift operator) for discrete-time systems. The uncertainty $\Delta$ is then assumed to belong to a set $\Delta$ defined as

$$
\Delta \triangleq\left\{\Delta \mid\left[\begin{array}{ccc}
A^{\Delta} & B_{\xi}^{\Delta} & B_{u}^{\Delta} \\
C_{z}^{\Delta} & D_{z \xi}^{\Delta} & D_{z u}^{\Delta} \\
C_{y}^{\Delta} & D_{y \xi}^{\Delta} & D_{y u}^{\Delta}
\end{array}\right] \in \mathcal{M}_{s y n}\right\}
$$

where $\mathcal{M}_{\text {syn }}$ is a given convex polypope of system matrices

$$
\mathcal{M}_{s y n} \triangleq \mathbf{c o}\left\{\left[\begin{array}{ccc}
A_{i} & B_{\xi, i} & B_{u, i} \\
C_{z, i} & D_{z \xi, i} & D_{z u, i} \\
C_{y, i} & D_{y \xi, i} & D_{y u, i}
\end{array}\right], i=1,2, \ldots N\right\} \text {. }
$$

Interconnected to system (1) is the following full-order dynamic output-feedback controller

$$
\mathcal{C}_{\sigma}:\left\{\begin{aligned}
\sigma x^{c} & =A_{c} x^{c}+B_{c} y \\
u & =F x^{c}
\end{aligned}\right.
$$

with $x^{c} \in \mathbb{R}^{n}$ its state. This yields the closed-loop system

$$
\left\{\begin{aligned}
\sigma \tilde{x} & =A_{c l}^{\Delta} \tilde{x}+B_{c l}^{\Delta} \xi \\
z & =C_{c l}^{\Delta} \tilde{x}+D_{c l}^{\Delta} \xi
\end{aligned}\right.
$$

where it is denoted $\tilde{x}^{T}=\left[x^{T},\left(x^{c}\right)^{T}\right]$, and

$$
\left[\begin{array}{c|c}
A_{c l}^{\Delta} & B_{c l}^{\Delta} \\
\hline C_{c l}^{\Delta} & D_{c l}^{\Delta}
\end{array}\right] \triangleq\left[\begin{array}{cc|c}
A^{\Delta} & B_{u}^{\Delta} F & B_{\xi}^{\Delta} \\
B_{c} C_{y}^{\Delta} & A_{c}+B_{c} D_{y u}^{\Delta} F & B_{c} D_{y \xi}^{\Delta} \\
\hline C_{z}^{\Delta} & D_{z u}^{\Delta} F & D_{z \xi}^{\Delta}
\end{array}\right] .
$$

Denoting the transfer function from the disturbance $\xi$ to the controlled output $z$, corresponding to the state-space model (5), as

$$
T_{c l}^{\Delta}(\sigma) \triangleq C_{c l}^{\Delta}\left(\sigma I_{2 n}-A_{c l}^{\Delta}\right)^{-1} B_{c l}^{\Delta}+D_{c l}^{\Delta},
$$

then this paper addresses the following problem: given positive scalars $\alpha_{2}$ and $\alpha_{\infty}$ and a convex set $\mathcal{D}$, defined as

$$
\mathcal{D} \triangleq\left\{z \in \mathbb{C}: L_{\mathcal{D}}+\operatorname{Sym}\left(z M_{\mathcal{D}}\right)<0\right\},
$$

for some given real matrices $L_{\mathcal{D}}=L_{\mathcal{D}}^{T}$ and $M_{\mathcal{D}}$, find constant matrices $A_{c}, B_{c}$, and $F$, parametrizing the controller (4), that solve the following constrained optimization problem

$$
\begin{aligned}
& \text { subject to: } \\
& \min _{\gamma_{2}, \gamma_{\infty}, A_{c}, B_{c}, F} \alpha_{2} \gamma_{2}+\alpha_{\infty} \gamma_{\infty} \\
& \mathcal{H}_{2} \text { objective: } \quad \sup _{\Delta}\left\|L_{2}\left(T_{c l}^{\Delta}(\sigma)-D_{c l}^{\Delta}\right) R_{2}\right\|_{2}^{2}<\gamma_{2}, \\
& \mathcal{H}_{\infty} \text { objective: } \quad \sup _{\infty \in \Delta}\left\|L_{\infty l} T_{c l}^{\Delta}(\sigma) R_{\infty}\right\|_{\infty}^{2}<\gamma_{\infty}, \\
& \text { Pole-placement: } \lambda\left(A_{c l}^{\Delta}\right) \in \mathcal{D}, \forall \Delta \in \Delta \text {. }
\end{aligned}
$$

where the matrices $L_{2}, R_{2}, L_{\infty}$, and $R_{\infty}$, are used to select the desired input-output channels that need to satisfy the required constraint in (9).

As discussed in the introduction, this problem is not convex and is NP-hard. In the next section we will present a new algorithm which can be used for finding a locally optimal solution to the problem defined in (9). As most local approaches, this approach requires an initially feasible solution from which the local optimization is initiated. For the purposes of its initialization, a computationally fast approach based on LMIs for finding an initially feasible controller is later on proposed in Sec. IV. A summary of the complete algorithm is given in Sec. V.

\section{DESIGN VIA BMI OPTIMIZATION}

Define the following $N$ biaffine functions

$$
\begin{aligned}
& \mathcal{B M I}^{(k)}(\boldsymbol{x}, \boldsymbol{y}) \triangleq \\
& F_{00}^{(k)}+\sum_{i=1}^{N_{1}} F_{i 0}^{(k)} \boldsymbol{x}_{i}+\sum_{j=1}^{N_{2}} F_{0 j}^{(k)} \boldsymbol{y}_{j}+\sum_{i=1}^{N_{1}} \sum_{j=1}^{N_{2}} F_{i j}^{(k)} \boldsymbol{x}_{i} \boldsymbol{y}_{j},
\end{aligned}
$$

where $F_{i j}^{(k)}=\left(F_{i j}^{(k)}\right)^{T}, i=0,1, \ldots, N_{1}, j=0,1, \ldots, N_{2}, k=$ $1, \ldots, N$ are given symmetric matrices. In this paper we consider the following BMI optimization problem

$$
(\mathcal{P}):\left\{\begin{array}{l}
\min \gamma, \text { over } \boldsymbol{x} \in \mathbb{R}^{N_{1}}, \boldsymbol{y} \in \mathbb{R}^{N_{2}}, \text { and } \gamma \in \mathbb{R} \\
\mathcal{B M}^{(k)}(\boldsymbol{x}, \boldsymbol{y}) \leq 0, \quad k=1,2, \ldots, N \\
\langle c, \boldsymbol{x}\rangle+\langle d, \boldsymbol{y}\rangle \leq \gamma .
\end{array}\right.
$$

This problem is known to be NP-hard [15].

It is a fact that for systems with polypotic uncertainty the outputfeedback controller design problem can be written as BMIs in the general form (11) [17].

Let us, for now, consider the feasibility problem for a fixed $\gamma$. Denote

$$
\mathcal{B M}^{(N+1)}(\boldsymbol{x}, \boldsymbol{y}) \triangleq\langle c, \boldsymbol{x}\rangle+\langle d, \boldsymbol{y}\rangle-\gamma .
$$

The feasibility problem is then defined as

$$
(\mathcal{F P}):\left\{\begin{array}{l}
\text { Find } \boldsymbol{x} \in \mathbb{R}^{N_{1}}, \boldsymbol{y} \in \mathbb{R}^{N_{2}} \\
\text { such that } \bigoplus_{k=1}^{N+1} \mathcal{B M I}^{(k)}(\boldsymbol{x}, \boldsymbol{y}) \leq 0 .
\end{array}\right.
$$

Define the following cost function

$$
v_{\gamma}(\boldsymbol{x}, \boldsymbol{y}) \triangleq\left\|\left[\bigoplus_{k=1}^{N+1} \mathcal{B} \mathcal{M} \mathcal{I}^{(k)}(\boldsymbol{x}, \boldsymbol{y})\right]^{+}\right\|_{F}^{2} \geq 0
$$

From the definition of the projection $[.]^{+}$, and from the properties of the Frobenius norm we can write

$$
v_{\gamma}(\boldsymbol{x}, \boldsymbol{y})=\sum_{k=1}^{N+1}\left\|\left[\mathcal{B M}^{(k)}(\boldsymbol{x}, \boldsymbol{y})\right]^{+}\right\|_{F}^{2} \triangleq \sum_{k=1}^{N+1} v_{\gamma}^{(k)}(\boldsymbol{x}, \boldsymbol{y}) .
$$


It is therefore clear that

$$
(\mathcal{F P}) \text { is feasible } \Leftrightarrow 0 \in \min _{\boldsymbol{x}, \boldsymbol{y}} v_{\gamma}(\boldsymbol{x}, \boldsymbol{y}) .
$$

In this way we have rewritten the initial BMI problem into an equivalent optimization problem. The goal is now to search for a local minimum of $v_{\gamma}$. However, the function $v_{\gamma}(\boldsymbol{x}, \boldsymbol{y})$ is not convex. Even worse, it may have multiple local minima. Now, if $\left(\boldsymbol{x}^{*}, \boldsymbol{y}^{*}\right)$ is a local minimum for $v_{\gamma}$ and is such that $v_{\gamma}\left(\boldsymbol{x}^{*}, \boldsymbol{y}^{*}\right)=0$, then $\left(\boldsymbol{x}^{*}, \boldsymbol{y}^{*}\right)$ is also a feasible solution to $(\mathcal{F P})$. However, if $\left(\boldsymbol{x}^{*}, \boldsymbol{y}^{*}\right)$ is such that $v_{\gamma}\left(\boldsymbol{x}^{*}, \boldsymbol{y}^{*}\right)>0$, then we cannot say anything about the feasibility of $(\mathcal{F P})$. The idea is then to start from a feasible solution for a given $\gamma$, and then apply the method of bisection over $\gamma$ to achieve a local minimum with a desired precision, at each iteration searching for a feasible solution to $(\mathcal{F P})$. This is described in more detail in Sec. V.

Let us now concentrate on the problem of finding a local solution to

$$
\min _{\boldsymbol{x}, \boldsymbol{y}} v_{\gamma}(\boldsymbol{x}, \boldsymbol{y})
$$

The goal is to develop an approach that has a guaranteed convergence to a local optimum of $v_{\gamma}(\boldsymbol{x}, \boldsymbol{y})$. To this end, we first note that the function $v_{\gamma}(\boldsymbol{x}, \boldsymbol{y})$ is differentiable, and we derive an expression for its gradient.

Theorem 1: Consider the function

$$
(f \circ G)(\boldsymbol{x}, \boldsymbol{y}) \triangleq\left\|[G(\boldsymbol{x}, \boldsymbol{y})]^{+}\right\|_{F}^{2},
$$

where $G: \mathbb{R}^{N_{1}} \times \mathbb{R}^{N_{2}} \mapsto \mathbb{R}^{q \times q}$ is defined as follows

$$
G(\boldsymbol{x}, \boldsymbol{y})=F_{00}+\sum_{i=1}^{N_{1}} F_{i 0} \boldsymbol{x}_{i}+\sum_{j=1}^{N_{2}} F_{0 j} \boldsymbol{y}_{j}+\sum_{i=1}^{N_{1}} \sum_{j=1}^{N_{2}} F_{i j} \boldsymbol{x}_{i} \boldsymbol{y}_{j}
$$

The function $(f \circ G)(\boldsymbol{x}, \boldsymbol{y})$ is differentiable, and

$$
\nabla(f \circ G)(\boldsymbol{x}, \boldsymbol{y}) \triangleq\left[\frac{\partial}{\partial x_{1}} \cdots \frac{\partial}{\partial x_{N_{1}}}, \frac{\partial}{\partial y_{1}} \ldots \frac{\partial}{\partial y_{N_{2}}}\right]^{T}(f \circ G)(\boldsymbol{x}, \boldsymbol{y}),
$$

with

$$
\begin{gathered}
\frac{\partial}{\partial \boldsymbol{x}_{i}}(f \circ G)(\boldsymbol{x}, \boldsymbol{y})=2\left\langle[G(\boldsymbol{x}, \boldsymbol{y})]^{+}, F_{i 0}+\sum_{j=1}^{N_{2}} F_{i j} \boldsymbol{y}_{j}\right\rangle, \\
\frac{\partial}{\partial \boldsymbol{y}_{j}}(f \circ G)(\boldsymbol{x}, \boldsymbol{y})=2\left\langle[G(\boldsymbol{x}, \boldsymbol{y})]^{+}, F_{0 j}+\sum_{i=1}^{N_{1}} F_{i j} \boldsymbol{x}_{i}\right\rangle,
\end{gathered}
$$

is its gradient.

Proof: Using the properties of the projection [.] ${ }^{+}$we infer for any matrices $G$ and $\Delta G$, that

$$
\begin{aligned}
& f \circ(G+\Delta G)=\left\|[G+\Delta G]^{+}\right\|_{F}^{2}=\left\|G+\Delta G-[G+\Delta G]^{-}\right\|_{F}^{2} \\
& =\min _{S}\|G+\Delta G-S\|_{F}^{2} \leq\left\|G+\Delta G-[G]^{-}\right\|_{F}^{2} \\
& =\left\|[G]^{+}+\Delta G\right\|_{F}^{2}=\left\|[G]^{+}\right\|_{F}^{2}+2\left\langle[G]^{+}, \Delta G\right\rangle+\|\Delta G\|_{F}^{2} .
\end{aligned}
$$

On the other hand,

$$
\begin{aligned}
& f \circ(G+\Delta G)=\left\|[G+\Delta G]^{+}\right\|_{F}^{2}=\left\|G+\Delta G-[G+\Delta G]^{-}\right\|_{F}^{2} \\
& =\left\|[G]^{+}+[G]^{-}+\Delta G-[G+\Delta G]^{-}\right\|_{F}^{2} \\
& \geq\left\|[G]^{+}\right\|_{F}^{2}+2\left\langle[G]^{+}, \Delta G\right\rangle+2\left\langle[G]^{+},[G]^{-}\right\rangle+2\left\langle[G]^{+},-[G+\Delta G]^{-}\right\rangle \\
& \geq\left\|[G]^{+}\right\|_{F}^{2}+2\left\langle[G]^{+}, \Delta G\right\rangle .
\end{aligned}
$$

Thus we have $f \circ(G+\Delta G)=f \circ G+2\left\langle[G]^{+}, \Delta G\right\rangle+O\left(\|\Delta G\|_{F}^{2}\right)$. Now, let $G(\boldsymbol{x}, \boldsymbol{y})$ be defined as in (15), and define

$$
\begin{aligned}
& \Delta G(\boldsymbol{x}, \boldsymbol{y})=G(\boldsymbol{x}+\Delta \boldsymbol{x}, \boldsymbol{y}+\Delta \boldsymbol{y})-G(\boldsymbol{x}, \boldsymbol{y}) \\
& =\sum_{i=1}^{N_{1}} \tilde{F}_{i 0} \Delta \boldsymbol{x}_{i}+\sum_{j=1}^{N_{2}} \tilde{F}_{0 j} \Delta \boldsymbol{y}_{j}+\sum_{i=1}^{N_{1}} \sum_{j=1}^{N_{2}} F_{i j} \Delta \boldsymbol{x}_{i} \Delta \boldsymbol{y}_{j},
\end{aligned}
$$

where it is denoted

$$
\tilde{F}_{i 0}=F_{i 0}+\sum_{j=1}^{N_{2}} F_{i j} \boldsymbol{y}_{j}, \quad \tilde{F}_{0 j}=F_{0 j}+\sum_{i=1}^{N_{1}} F_{i j} \boldsymbol{x}_{i} .
$$

Then

$$
\begin{aligned}
& (f \circ G)(\boldsymbol{x}+\Delta \boldsymbol{x}, \boldsymbol{y}+\Delta \boldsymbol{y})=(f \circ G)(\boldsymbol{x}, \boldsymbol{y}) \\
& +2 \sum_{i=1}^{N_{1}}\left\langle[G(\boldsymbol{x}, \boldsymbol{y})]^{+}, \tilde{F}_{i 0}\right\rangle \Delta \boldsymbol{x}_{\boldsymbol{i}}+2 \sum_{j=1}^{N_{2}}\left\langle[G(\boldsymbol{x}, \boldsymbol{y})]^{+}, \tilde{F}_{0 j}\right\rangle \Delta \boldsymbol{y}_{j} \\
& +2 \sum_{i=1}^{N_{1}} \sum_{j=1}^{N_{2}}\left\langle[G(\boldsymbol{x}, \boldsymbol{y})]^{+}, F_{i j} \Delta \boldsymbol{x}_{\boldsymbol{i}} \Delta \boldsymbol{y}_{j}\right\rangle+O\left(\left\|(\Delta \boldsymbol{x}, \Delta \boldsymbol{y})^{2}\right\|\right) .
\end{aligned}
$$

It is thus clear that $f$ is differentiable and its partial derivatives are given by the expressions (16) and (17).

The partial derivatives of our original function $v_{\gamma}(\boldsymbol{x}, \boldsymbol{y})$ can then be directly derived using the result of Theorem 1 :

$$
\begin{aligned}
& \frac{\partial v_{\gamma}(\boldsymbol{x}, \boldsymbol{y})}{\partial \boldsymbol{x}_{i}}=2 \sum_{k=1}^{N+1}\left\langle\left[\mathcal{B M} \mathcal{I}^{(k)}(\boldsymbol{x}, \boldsymbol{y})\right]^{+}, F_{i 0}^{(k)}+\sum_{j=1}^{N_{2}} F_{i j}^{(k)} \boldsymbol{y}_{j}\right\rangle \\
& \frac{\partial v_{\gamma}(\boldsymbol{x}, \boldsymbol{y})}{\partial \boldsymbol{y}_{j}}=2 \sum_{k=1}^{N+1}\left\langle\left[\mathcal{B M} \mathcal{I}^{(k)}(\boldsymbol{x}, \boldsymbol{y})\right]^{+}, F_{0 j}^{(k)}+\sum_{i=1}^{N_{1}} F_{i j}^{(k)} \boldsymbol{x}_{i}\right\rangle
\end{aligned}
$$

Note that these partial derivatives are continuous functions, so that $v_{\gamma} \in C^{1}$. Note also, that a lower bound on the cost function in (11) can always be obtained by solving the so-called relaxed LMI optimization problem [16]

$$
\begin{aligned}
& \gamma_{L B}=\min _{\boldsymbol{x}, \boldsymbol{y}}\langle c, \boldsymbol{x}\rangle+\langle d, \boldsymbol{y}\rangle, \\
& F_{00}^{(k)}+\sum_{i=1}^{N_{1}} F_{i 0}^{(k)} \boldsymbol{x}_{i}+\sum_{j=1}^{N_{2}} F_{0 j}^{(k)} \boldsymbol{y}_{j}+\sum_{i=1}^{N_{1}} \sum_{j=1}^{N_{2}} F_{i j}^{(k)} \boldsymbol{w}_{i j} \leq 0, \quad \forall k .
\end{aligned}
$$

If this problem is not feasible, then the original BMI problem is also not feasible.

Now that it was shown that the function $v_{\gamma} \in C^{1}$ and an expression for its gradient has been derived, the cautious BFGS quasi-Newton type of optimization algorithm, adopted from [11], can be used for finding a local minimum of $v_{\gamma} \in C^{1}$. Assuming that the level set $\left.\Omega=\left\{\boldsymbol{x}, \boldsymbol{y}: v_{\gamma}(\boldsymbol{x}, \boldsymbol{y}) \leq v_{\gamma}\left(\boldsymbol{x}^{(\mathbf{0})}, \boldsymbol{y}^{(0)}\right)\right)\right\}$ is compact, the convergence of this algorithm is established in [11] provided that the following conditions hold: (a) $v_{\gamma}(\boldsymbol{x}, \boldsymbol{y})$ is continuously differentiable on $\Omega$ with gradient denoted as $g(\boldsymbol{x}, \boldsymbol{y})$, and (b) there exists a constant $L>0$ such that the global Lipschitz condition holds:

$\|g(\boldsymbol{x}, \boldsymbol{y})-g(\overline{\boldsymbol{x}}, \overline{\boldsymbol{y}})\|_{2} \leq L\left\|\left[\begin{array}{l}\boldsymbol{x} \\ \boldsymbol{y}\end{array}\right]-\left[\begin{array}{c}\overline{\boldsymbol{x}} \\ \overline{\boldsymbol{y}}\end{array}\right]\right\|_{2}, \forall(\boldsymbol{x}, \boldsymbol{y}),(\overline{\boldsymbol{x}}, \overline{\boldsymbol{y}}) \in \Omega$.

Condition (a) was shown in Theorem 1. Condition (b) follows by observing that the projection [.] ${ }^{+}$is Lipschitz, and since $\mathcal{B M I}^{(k)}(\boldsymbol{x}, \boldsymbol{y})$ is smooth the functions in (18) and (19) satisfy a local Lipschitz condition. The compactness of the set $\Omega$ then implies the global Lipschitz condition.

In the next section we focus on the problem of finding an initial feasible solution to the BMI optimization problem.

\section{INITIAL CONTROLLER DESIGN}

In this Section, a two-step procedure is presented for the design of an initial feasible robust output-feedback controller. It consists of two steps. In the first step a robust state-feedback gain matrix $\boldsymbol{F}$ is designed such that the multiobjective criterion of the form (9) is satisfied for the closed-loop system with state-feedback control $u=F x$. This problem is convex and is considered in Subsection 
Sec. IV-A. In the second step the computed state-feedback gain matrix $F$ is plugged into the original closed-loop system (5), and the multiobjective control problem, defined in (9), is solved in terms of the remaining unknown controller matrices $\boldsymbol{A}_{\boldsymbol{c}}$ and $\boldsymbol{B}_{\boldsymbol{c}}$. This problem is discussed in Sec. IV-B.

Before we present the solutions to these problem we summarize the following standard results, adopted from [12], [4], [2].

Lemma 1: Let $\mathcal{A}$ be a real matrix. Then $\lambda(\mathcal{A}) \in \mathcal{D}$, with $\mathcal{D}$ defined in (8), if and only if there exists a matrix $\boldsymbol{P}=\boldsymbol{P}^{T}>0$ such that $L_{\mathcal{D}} \otimes \boldsymbol{P}+\operatorname{Sym}\left(M_{\mathcal{D}} \otimes(\boldsymbol{P} \mathcal{A})\right)<0$.

Define

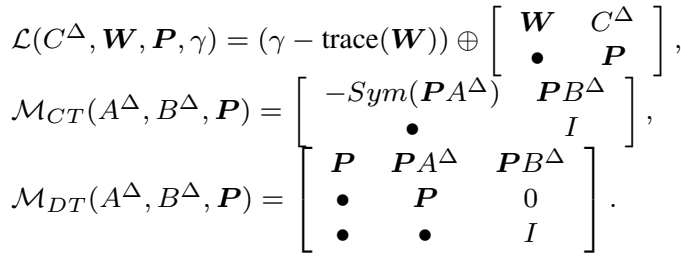

Lemma $2\left(\mathcal{H}_{2}\right.$ norm): Assume that $D^{\Delta}=0$. Then $\sup _{\Delta \in \Delta}\left\|T_{c l}^{\Delta}(\sigma)\right\|_{2}^{2}<\gamma$ if there exist matrices $\boldsymbol{P}=\boldsymbol{P}^{T}$ and $\boldsymbol{W}=\boldsymbol{W}^{T}$ such that for all $\Delta \in \Delta$

$\mathcal{L}\left(C_{c l}^{\Delta}, \boldsymbol{W}, \boldsymbol{P}, \gamma\right) \oplus \mathcal{M}_{C T}\left(A_{c l}^{\Delta}, B_{c l}^{\Delta}, \boldsymbol{P}\right)>0$, (cont. case),

$\mathcal{L}\left(C_{c l}^{\Delta}, \boldsymbol{W}, \boldsymbol{P}, \gamma\right) \oplus \mathcal{M}_{D T}\left(A_{c l}^{\Delta}, B_{c l}^{\Delta}, \boldsymbol{P}\right)>0$, (discr. case).

Lemma $3\left(\mathcal{H}_{\infty}\right.$ norm): $\sup _{\Delta \in \Delta}\left\|T_{c l}^{\Delta}(\sigma)\right\|_{\infty}^{2}<\gamma$ if there exists a matrix $\boldsymbol{P}=\boldsymbol{P}^{T}$ such that for all $\Delta \in \boldsymbol{\Delta}$

$\boldsymbol{P} \oplus\left[\begin{array}{ccc}\mathcal{M}_{C T}\left(A_{c l}^{\Delta}, B_{c l}^{\Delta}, \boldsymbol{P}\right) & {\left[\begin{array}{cc}C_{c l}^{\Delta}, & D_{c l}^{\Delta}\end{array}\right]^{T}}\end{array}\right]>0$, (cont. case),
$\left[\begin{array}{ccc}\mathcal{M}_{D T}\left(A_{c l}^{\Delta}, B_{c l}^{\Delta}, \boldsymbol{P}\right) & {\left[\begin{array}{ccc}0, & C_{c l}^{\Delta}, & D_{c l}^{\Delta}\end{array}\right]^{T}}\end{array}\right]>0$, (discr. case).

\section{A. Step 1: Robust Multiobjective State-Feedback Design}

The state-feedback case the for system (1) is equivalent to taking $C_{y}^{\Delta}=I_{n}, D_{y \xi}^{\Delta}=0_{n \times n_{\xi}}, D_{y u}^{\Delta}=0_{n \times m}$, so that $y \equiv x$. Furthermore, we consider the constant state-feedback controller $u=F x$, which results in the closed-loop transfer function

$$
\tilde{T}_{c l}^{\Delta}(\sigma) \triangleq\left(C_{z}^{\Delta}+D_{z u}^{\Delta} F\right)\left(\sigma I_{n}-\left(A^{\Delta}+B_{u}^{\Delta} F\right)\right)^{-1} B_{\xi}^{\Delta}+D_{z \xi}^{\Delta} .
$$

The following Theorem can be used for robust multiobjective state-feedback design for discrete-time and continuous-time systems. The proof is based on [13], [12] and follows after rewriting Lemmas 2 and 3 for the closed-loop system (22) as LMIs in $\boldsymbol{Q}=\boldsymbol{P}^{-1}$, with subsequent change of variables.

Theorem 2 (Robust Multiobjective State-Feedback Control): Consider the system (1), and assume that $C_{y}^{\Delta}=I_{n}, D_{y \xi}^{\Delta}=0_{n \times n_{\xi}}$, $D_{y u}^{\Delta}=0_{n \times m}$. Consider the controller $u=F x$ resulting in the closed-loop transfer function $\tilde{T}_{c l}^{\Delta}(\sigma)$, defined in (22). Given matrices $L_{2}, R_{2}, L_{\infty}$, and $R_{\infty}$, the conditions

$$
\begin{aligned}
& \sup _{\Delta \in \Delta}\left\|L_{2}\left(\tilde{T}_{c l}^{\Delta}(\sigma)-D_{z \xi}^{\Delta}\right) R_{2}\right\|_{2}^{2}<\gamma_{2}, \\
& \sup _{\Delta \in \Delta}\left\|L_{\infty} \tilde{T}_{c l}^{\Delta}(\sigma) R_{\infty}\right\|_{\infty}^{2}<\gamma_{\infty}, \\
& \lambda\left(A^{\Delta}+B_{u}^{\Delta} F\right) \in \mathcal{D}, \forall \Delta \in \boldsymbol{\Delta} .
\end{aligned}
$$

hold if there exist matrices $\boldsymbol{Q}=\boldsymbol{Q}^{T}, \boldsymbol{W}=\boldsymbol{W}^{T}, \boldsymbol{R}=\boldsymbol{R}^{T}$, and
$\boldsymbol{L}$ such that for all $i=1, \ldots, N$ the following LMIs hold

$$
\begin{aligned}
& \text { PP: }(-\boldsymbol{Q}) \oplus\left(L_{\mathcal{D}} \otimes \boldsymbol{Q}+\operatorname{Sym}\left(M_{\mathcal{D}} \otimes \boldsymbol{\Sigma}_{i}\right)\right)<0, \\
& \mathcal{H}_{2}:\left(\gamma_{2}-\operatorname{trace}(\boldsymbol{R})\right) \oplus\left[\begin{array}{cc}
\boldsymbol{R} & L_{2} \boldsymbol{\Omega}_{i} \\
\bullet & \boldsymbol{Q}
\end{array}\right] \\
& \oplus\left\{\begin{array}{cc}
-\operatorname{Sym}\left(\boldsymbol{\Sigma}_{i}\right) & B_{\xi, i} R_{2} \\
\dot{\bullet} & I
\end{array}\right]>0, \text { (continuous case) } \\
& \oplus\left\{\begin{array}{ccc}
\boldsymbol{Q} & \boldsymbol{\Sigma}_{i} & B_{\xi, i} R_{2} \\
\bullet & \boldsymbol{Q} & 0 \\
\bullet & \bullet & I
\end{array}\right]>0 \text {, (discrete case) }
\end{aligned}
$$

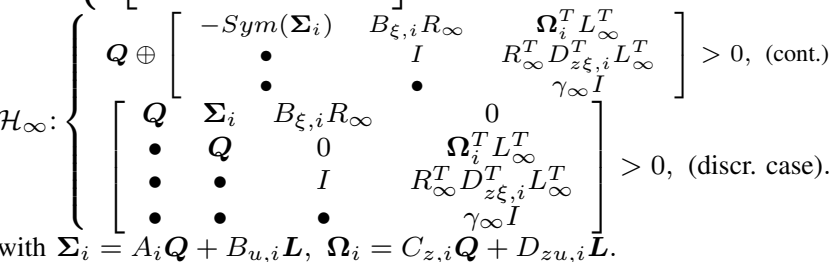

The state-feedback gain matrix $F$ is then given by $F=\boldsymbol{L} \boldsymbol{Q}^{-1}$.

\section{B. Step 2: Robust Multiobjective Output-Feedback Design}

In what follows we assume that the optimal state-feedback gain $F$ has already been computed at Step 1. In contrast to Step 1, the problem defined in Step 2 of the algorithm at the beginning of Sec. IV is certainly non-convex in the variables $\boldsymbol{P}, \boldsymbol{W}, \boldsymbol{A}_{\boldsymbol{c}}$, and $\boldsymbol{B}_{\boldsymbol{c}}$ since application of Lemmas 2 and 3 to the closed-loop system in (6) leads to non-linear matrix inequalities due to the fact that the variables $\boldsymbol{A}_{\boldsymbol{c}}$ and $\boldsymbol{B}_{\boldsymbol{c}}$ appear in the closed-loop system matrices $\boldsymbol{A}_{c l}^{\Delta}$ and $\boldsymbol{B}_{c l}^{\Delta}$ (for which reason the last two are typed in boldface). However, by introducing some conservatism by means of constraining the Lyapunov matrix $\boldsymbol{P}$ to have block-diagonal structure

$$
\boldsymbol{P}=\boldsymbol{X} \oplus \boldsymbol{Y}
$$

the nonlinear matrix inequalities in question can be written as LMIs. However, it can easily be seen that a necessary condition for the existence of a structured Lyapunov matrix of the form (23) for $\boldsymbol{A}_{c l}^{\Delta}$ defined in (6) is that the matrix $A^{\Delta}$ is stable for all $\Delta \in \Delta$. Luckily, this restriction can be removed by introducing a change of basis of the state vector of the closed-loop system to $\bar{x}=\left[x^{T}, x^{T}-\left(x^{c}\right)^{T}\right]^{T}$. For the resulting closed-loop system the structured Lyapunov matrix $\boldsymbol{P}$ does not impose the restriction for stability of $A^{\Delta}$. This is summarized in the following result.

Theorem 3 (Robust Multiobjective Output-Feedback Control): Consider the closed-loop system (5), with transfer function $T_{c l}^{\Delta}(\sigma)$ defined in (7), formed by interconnecting the plant (1) with the dynamic output-feedback controller (4), in which the state-feedback gain matrix $F$ is given. Then given matrices $L_{2}, R_{2}, L_{\infty}$, and $R_{\infty}$ of appropriate dimensions, the conditions

$$
\begin{aligned}
& \sup _{\Delta \in \Delta}\left\|L_{2}\left(T_{c l}^{\Delta}(\sigma)-D_{c l}^{\Delta}\right) R_{2}\right\|_{2}^{2}<\gamma_{2}, \\
& \sup _{\Delta \in \Delta}\left\|L_{\infty} T_{c l}^{\Delta}(\sigma) R_{\infty}\right\|_{\infty}^{2}<\gamma_{\infty}, \\
& \lambda\left(A_{c l}^{\Delta}\right) \in \mathcal{D}, \forall \Delta \in \Delta .
\end{aligned}
$$

hold if there exist matrices $\boldsymbol{W}=\boldsymbol{W}^{T}, \boldsymbol{X}=\boldsymbol{X}^{T}, \boldsymbol{Y}=\boldsymbol{Y}^{T}$, $\boldsymbol{Z}$ and $\boldsymbol{G}$ such that the following system of LMIs has a feasible 
solution for all $i=1, \ldots, N$

$$
\begin{aligned}
& \text { PP: }(-\boldsymbol{P}) \oplus\left(L_{\mathcal{D}} \otimes \boldsymbol{P}+\operatorname{Sym}\left(M_{\mathcal{D}} \otimes \boldsymbol{M}_{i}\right)\right)<0, \\
& \mathcal{H}_{2}:\left(\gamma_{2}-\operatorname{trace}(\boldsymbol{W})\right) \oplus\left[\begin{array}{cc}
\boldsymbol{W} & L_{2} \bar{C}_{c l, i} \\
\bullet & \boldsymbol{P}
\end{array}\right] \\
& \oplus\left\{\begin{array}{ccc}
-\operatorname{Sym}\left(\boldsymbol{M}_{i}\right) & \boldsymbol{N}_{i} R_{2} \\
\bullet & I
\end{array}\right]>0, \text { (cont. case) }
\end{aligned}
$$

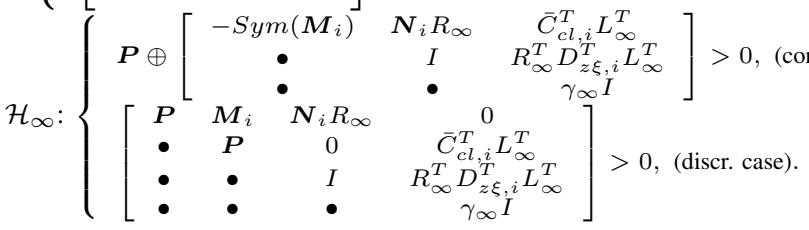

where the matrices $\boldsymbol{M}_{i}, \boldsymbol{N}_{i}, R$, and $\boldsymbol{P}$ are defined as

$$
\begin{gathered}
\boldsymbol{M}_{i}=\left[\begin{array}{c}
\boldsymbol{X}\left(A_{i}+B_{u, i} F\right) \\
\boldsymbol{Y}\left(A_{i}+B_{u, i} F\right)-\boldsymbol{Z}-\boldsymbol{G}\left(C_{y, i}+D_{y u, i} F\right) \\
-\boldsymbol{X} B_{u, i} F \\
\boldsymbol{Z}+\boldsymbol{G} D_{y u, i} F-\boldsymbol{Y} B_{u, i} F
\end{array}\right], \\
\boldsymbol{N}_{i}=\left[\begin{array}{c}
\boldsymbol{X} B_{\xi, i} \\
\boldsymbol{Y} B_{\xi, i}-\boldsymbol{G} D_{y \xi, i}
\end{array}\right], \boldsymbol{P}=\left[\begin{array}{cc}
\boldsymbol{X} \\
& \boldsymbol{Y}
\end{array}\right], \\
\bar{C}_{c l, i}=\left[\begin{array}{cc}
C_{z, i}+D_{z u, i} F, & -D_{z u, i} F
\end{array}\right] .
\end{gathered}
$$

Furthermore, the unknown matrices $\boldsymbol{A}_{c}$ and $\boldsymbol{B}_{c}$ of the controller (4) are given by $\boldsymbol{A}_{\boldsymbol{c}}=\boldsymbol{Y}^{-1} \boldsymbol{Z}$ and $\boldsymbol{B}_{c}=\boldsymbol{Y}^{-1} \boldsymbol{G}$.

Proof: For the sake of brevity, only an outline of the proof is given. Let the $\overline{\boldsymbol{A}}_{c l}^{\Delta}, \overline{\boldsymbol{B}}_{c l}^{\Delta}, \bar{C}_{c l}^{\Delta}$, and $\bar{D}_{c l}^{\Delta}$, denote the closed-loop system matrices obtained after performing the change of basis of the state vector $\bar{x}=\left[x^{T}, x^{T}-\left(x^{c}\right)^{T}\right]^{T}$. Then application of Lemmas 2 and 3 to this transformed closed-loop system results in the bilinear terms $\boldsymbol{P} \overline{\boldsymbol{A}}_{c l}^{\Delta}$, and $\boldsymbol{P} \overline{\boldsymbol{B}}_{c l}^{\Delta}$ from the matrices $\mathcal{M}_{C T}\left(\overline{\boldsymbol{A}}_{c l}^{\Delta}, \overline{\boldsymbol{B}}_{c l}^{\Delta}, \boldsymbol{P}\right)$ and $\mathcal{M}_{D T}\left(\overline{\boldsymbol{A}}_{c l}^{\Delta}, \overline{\boldsymbol{B}}_{c l}^{\Delta}, \boldsymbol{P}\right)$, defined in (21). Clearly, with $\boldsymbol{P}$ defined as in (23) we can write

$$
\begin{aligned}
& \boldsymbol{P} \bar{A}_{c l}^{\Delta}= {\left[\begin{array}{c}
\boldsymbol{X}\left(A^{\Delta}+B_{u}^{\Delta} F\right) \\
\boldsymbol{Y}\left(A^{\Delta}+B_{u}^{\Delta} F\right)-\boldsymbol{Y} \boldsymbol{B}_{c}\left(C_{y}^{\Delta}+D_{y u}^{\Delta} F\right)-\boldsymbol{Y} \boldsymbol{A}_{c} \\
-\boldsymbol{X} B_{u}^{\Delta} F \\
\boldsymbol{Y} \boldsymbol{A}_{\boldsymbol{c}}+\boldsymbol{Y} \boldsymbol{B}_{\boldsymbol{c}} D_{y u}^{\Delta} F-\boldsymbol{Y} B_{u}^{\Delta} F
\end{array}\right], } \\
& \boldsymbol{X} B_{\xi}^{\Delta} \\
& \boldsymbol{P} \bar{B}_{c l}^{\Delta}=\left[\begin{array}{c}
\boldsymbol{Y} B_{\xi}^{\Delta}-\boldsymbol{Y} \boldsymbol{B}_{\boldsymbol{c}} D_{y \xi}^{\Delta}
\end{array}\right] .
\end{aligned}
$$

Making the one-to-one change of variables $\left[\begin{array}{lll}\boldsymbol{Y} \boldsymbol{A}_{\boldsymbol{c}} & \boldsymbol{Y} \boldsymbol{B}_{\boldsymbol{c}}\end{array}\right]=$ $\left[\begin{array}{ll}\boldsymbol{Z} & \boldsymbol{G}\end{array}\right]$ results in $\boldsymbol{P} \overline{\boldsymbol{A}}_{c l, i}=\boldsymbol{M}_{i}$, and $\boldsymbol{P} \overline{\boldsymbol{B}}_{c l, i}=\boldsymbol{N}_{i}$, with the matrices $\boldsymbol{M}_{i}$ and $\boldsymbol{N}_{i}$ defined as in (25), being linear in the new variables.

\section{SUMMARY OF THE APPROACH}

We next summarize the proposed approach to robust dynamic output-feedback controller design.

Algorithm 1 (Robust Output-Feedback Controller Design):

Use the result in Theorem 3 to find an initially feasible controller, represented by the variables $\left(\boldsymbol{x}_{0}, \boldsymbol{y}_{0}, \gamma_{0}\right)$ related to the corresponding BMI problem (11). Set $\left(\boldsymbol{x}^{*}, \boldsymbol{y}^{*}, \gamma_{U B}^{(0)}\right)=$ $\left(\boldsymbol{x}_{0}, \boldsymbol{y}_{0}, \gamma_{0}\right)$. Solve the relaxed LMI problem (20) to obtain $\gamma_{L B}^{(0)}$. Select the desired precision (relative tolerance) TOL and the maximum number of iterations allowed $k_{\max }$. Set $k=0$.

Step 1. TAKE $\gamma_{k}=\frac{\gamma_{U B}^{(k)}+\gamma_{L B}^{(k)}}{2}$, AND SOLVE THE PROBLEM $\left(\boldsymbol{x}_{k}, \boldsymbol{y}_{k}\right)=\arg \min v_{\gamma_{k}}(\boldsymbol{x}, \boldsymbol{y})$ STARTING WITH INITIAL CONDITION $\left(\boldsymbol{x}^{*}, \boldsymbol{y}^{*}\right)$.

Step 2. IF $v_{\gamma_{k}}\left(\boldsymbol{x}_{k}, \boldsymbol{y}_{k}\right)=0$ THEN SET $\left(\boldsymbol{x}^{*}, \boldsymbol{y}^{*}, \gamma_{U B}^{(k)}\right)=$ $\left(\boldsymbol{x}_{k}, \boldsymbol{y}_{k}, \gamma_{k}\right)$ ELSE SET $\gamma_{L B}^{(k)}=\gamma_{k}$.
Step 3. IF $\left|\gamma_{U B}^{(k)}-\gamma_{L B}^{(k)}\right|<\operatorname{TOL}\left|\gamma_{U B}^{(k)}\right|$ OR $k \geq k_{\text {max }}$ THEN STOP $\left(\left(\boldsymbol{x}^{*}, \boldsymbol{y}^{*}, \gamma_{U B}^{(k)}\right)\right.$ IS THE BEST FEASIBLE SOLUTION WITH THE DESIRED TOLERANCE) ELSE SET $k \leftarrow$ $k+1$ AND GO TO STEP 1 .

Note, that $\gamma_{L B}$ at each iteration represents an infeasible value for $\gamma$, while $\gamma_{U B}$ represents a feasible one. At each iteration of the algorithm the distance between these two bounds is reduced in two.

\section{CASE STUDY}

The example considered consists of a linear model of one joint of a real-life space robot manipulator (SRM) system. A continuous state-space model of the system is given by

$$
\begin{aligned}
\dot{x} & =\left[\begin{array}{cccc}
0 & 1 & 0 & 0 \\
0 & 0 & \frac{c}{N^{2} I_{m}} & 0 \\
0 & 0 & 0 & 1 \\
0 & -\frac{\beta}{I_{\text {son }}} & -\frac{c}{N^{2} I_{m}}-\frac{c}{I_{\text {son }}} & -\frac{\beta}{I_{\text {son }}}
\end{array}\right] x+\left[\begin{array}{c}
0 \\
\frac{K_{t}}{N I_{m}} \\
0 \\
-\frac{K_{t}}{N I_{m}}
\end{array}\right] u \\
y & =\left[\begin{array}{cccc}
1 & 0 & 1 & 0 \\
0 & N & 0 & 0
\end{array}\right] x,
\end{aligned}
$$

where $x=[\Omega, \dot{\Omega}, \epsilon, \dot{\epsilon}]^{T}$ is the state, and $u=i_{c}(\mathrm{~A})$ is the input. The system parameters are given in Table I. The damping coefficient $\beta$ and the spring constant $c$ are considered uncertain.

\begin{tabular}{|l|l|l|}
\hline Parameter: & Sym. & Value: \\
\hline \hline gearbox ratio & $N$ & -260.6 \\
\hline joint angle of inertial axis & $\Omega$ & variable \\
\hline effective joint input torque & $T_{j}^{\text {eff }}$ & variable \\
\hline motor torque constant & $K_{t}$ & 0.6 \\
\hline the damping coefficient & $\beta$ & {$[0.36,0.44]$} \\
\hline deformation torque of the gearbox & $T_{\text {def }}$ & variable \\
\hline inertia of the input axis & $I_{m}$ & 0.0011 \\
\hline inertia of the output system & $I_{s o n}$ & 400 \\
\hline joint angle of the output axis & $\epsilon$ & variable \\
\hline motor current & $i_{c}$ & variable \\
\hline spring constant & $c$ & {$\left[1.17 \times 10^{5}, 1.43 \times 10^{5}\right]$} \\
\hline
\end{tabular}

TABLE I

PARAMETERS IN THE MODEL OF SRM.

The objective is to find a controller that achieves for all possible values of the uncertain parameters a disturbance rejection of at least 1:100 for constant disturbances on the shaft angular position of the motor (such as, e.g., load), and a bandwidth of at least 1 [rad/sec]. This can be achieved by selecting the following performance weighting function (see the upper curve on Fig. 1 (bottom))

$$
W_{p}(s)=\frac{1}{s+0.01},
$$

and then requiring that $\left\|W_{p}(s) S(s)\right\|_{\infty}<1$, for all $\Delta \in \boldsymbol{\Delta}$, where $S(s)$ is the transfer function from the disturbance $d$ to the angular velocity $y_{2}=N \dot{\Omega}$. In other words, the design specifications would be achieved with a given controller $K(s)$ if the closed-loop transfer function from the disturbance $d$ to the controlled output $y_{2}$ lies below $W_{p}^{-1}(s)$ in the magnitude plot.

It should be noted here that this problem is of a rather large scale: the BMI optimization problem (11) consists of 4 bilinear matrix inequalities, each of dimension $12 \times 12$, and each a function of 95 variables (40 for the controller parameters, and 55 for the closedloop Lyapunov matrix). Also note, that the number of complicating variables, defined in [16] as $\min \{\operatorname{dim}(\boldsymbol{x}), \operatorname{dim}(\boldsymbol{y})\}$, in this example equals 40 . This makes it clear that the problem is far beyond the capabilities of the global approaches to solving the underlying BMI problem, which can at present deal with no more than just a few complicating variables. 

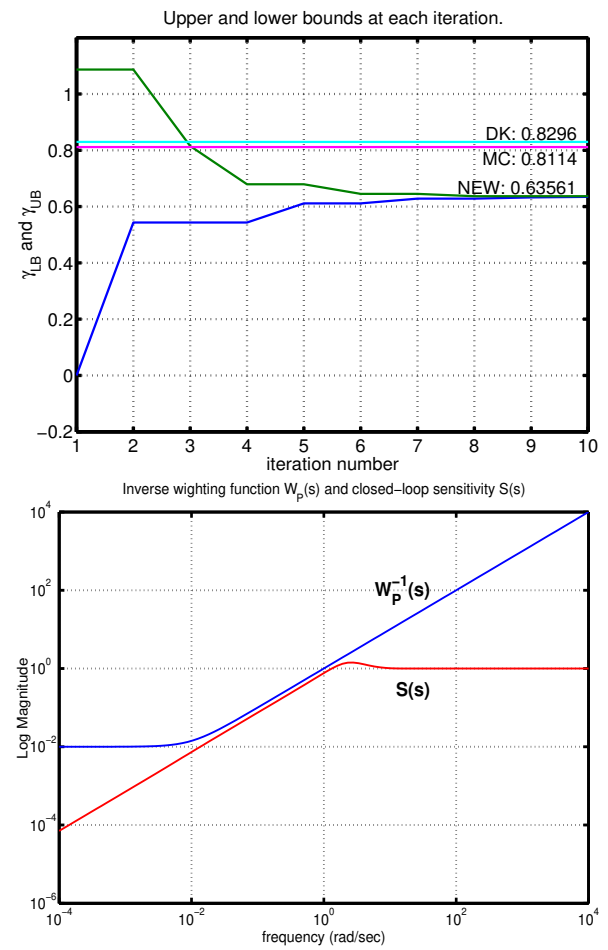

Fig. 1. Left: Upper and lower bounds on $\gamma$ during the BMI optimization. Right: Sensitivity function of the closed-loop system for the nominal values of the parameters and the inverse of the weighting function $W_{p}$.

First, using the result in Theorem 3 an initial controller was found achieving an upper bound of $\gamma_{\infty, \text { init }}=1.0866$, which was subsequently used to initialize the newly proposed BMI optimization (see Algorithm 1). The tolerance of TOL $=10^{-3}$ was selected, and $\gamma_{L B}^{(0)}=0$ since we know that $\gamma>0$ for the problem at hand. The new algorithm converged in 10 iterations to $\gamma_{\infty, N E W}=0.6356$. The computation took about 100 minutes on a PC with Intel(R) Pentium IV CPU $1500 \mathrm{MHz}$ and $1 \mathrm{~Gb}$ RAM.

Next, four other algorithms were tested on this example with the same initial controller, the same tolerance and the same stopping conditions. These algorithms were Rank Minimization Approach (RMA) [8], the Method of Centers (MC) [5], the Path-Following Method (PATH) [7], and the Alternating coordinate method (DK) [9]. From among these four approaches only two were able to improve the initial controller, namely the MC which achieved $\gamma_{\infty, M C}=0.8114$ in about 610 minutes. It was unable to improve the the performance further due to numerical problems. Similar problems were also reported in [3]. The DK iteration method terminated in about 20 minutes with $\gamma_{\infty, D K}=0.8296$. The PFM converged to an infeasible solution due to the fact that the initial condition is not "close enough" to the optimal one, so that the first order approximation that is made at each iteration is not accurate. Finally, the RMA method was also unable to find a feasible solution.

For the newly proposed method, the upper and the lower bounds on $\gamma$ at each iteration are plotted in Fig. 1 (top). Note that at each iteration the upper bound represents a feasible value for $\gamma$, and the lower bound - an infeasible one. Also plotted on the same figure are the values achieved by the DK iteration and the MC methods. With the optimal controller obtained with the newly proposed approach, the closed-loop sensitivity function is depicted in Fig. 1 (bottom), together with the inverse of the selected performance weighting function $W_{p}^{-1}(s)$. It can be seen from the figure that the sensitivity function remains below $W_{p}^{-1}(s)$, implying that the desired robust performance has been achieved.

\section{CONCLUSIONS}

In this paper a new approach to the design of locally optimal robust dynamic output-feedback controllers for systems with structured uncertainties was presented. The uncertainty is allowed to have a very general structure and is only assumed to be such that the state-space matrices of the system belong to a certain convex set. The approach is based on BMI optimization that is guaranteed to converge to a locally optimal solution provided that an initially feasible controller is given. This algorithm enjoys the useful properties of computational efficiency and guaranteed convergence to a local optimum. An algorithm for fast computation of an initially feasible controller is also provided and is based on a two-step procedure, where at each step an LMI optimization problem is solved - one to find the optimal state-feedback gain and one to find the remaining state-space matrices of the output-feedback controller. The design objectives considered are $\mathcal{H}_{2}, \mathcal{H}_{\infty}$, and pole-placement in LMI regions. The approach was tested on a model of one joint of a real-life space robotic manipulator, for which a robust $\mathcal{H}_{\infty}$ controller was designed.

\section{REFERENCES}

[1] G. Calafiore and B. Polyak. Stochastic algorithms for exact and approximate feasibility of robust LMIs. IEEE Transactions on Automatic Control, 46(11):17551759, 2001.

[2] M. Chilali, P. Gahinet, and P. Apkarian. Robust pole placement in LMI regions. IEEE Trans. on Automatic Control, 44(12):2257-2269, 1999.

[3] M. Fukuda and M. Kojima. Branch-and-cut algorithms for the bilinear matrix inequality eigenvalue problem. Computational Optimization and Applications, 19:79-105, 2001.

[4] J.C. Geromel and M.C. Oliveira. $\mathcal{H}_{2}$ and $\mathcal{H}_{\infty}$ robust filtering for convex bounded uncertain systems. IEEE Transactions on Automatic Control, 46(1):100$107,2001$.

[5] K.-C. Goh, M.G. Safonov, and G.P. Papavassilopoulos. A global optimization approach for the BMI problem. In Proceedings of the 33rd Conference on Decision and Control, pages 2009-2014, Lake Buena Vista, FL, 1994.

[6] K. Grigoradis and R. Skelton. Low-order control design for LMI problems using alternating projection methods. Automatica, 32(8):1117-1125, 1996.

[7] A. Hassibi, J. How, and S. Boyd. A path-following method for solving BMI problems in control. In Proceedings of the American Control Conference, pages 1385-1389, San Diego, CA, 1999.

[8] S. Ibaraki and M. Tomizuka. Rank minimization approach for solving BMI problems with random search. In Proceedings of the American Control Conference, pages 1870-1875, Arlington, VA, 2001.

[9] T. Iwasaki. The dual iteration for fixed order control. IEEE Transations on Automatic Control, 44(4):783-788, 1999.

[10] T. Iwasaki and R.E. Skelton. The XY-centering algorithm for the dual LMI problem: A new approach to fixed order control design. International Journal of Control, 62(6):1257-1272, 1995.

[11] D.H. Li and M. Fukushima. On the convergence of the BFGS method for nonconvex unconstrained optimization problems. SIAM Journal on Optimization, 11(4):1054-1064, 2001.

[12] M.C. Oliveira, J.C. Geromel, and J. Bernussou. An LMI optimization approach to multiobjective controller design for discrete-time systems. In Proc. of the IEEE Conference on Decision and Control, pages 3611-3616, Phoenix, Arizona, 1999.

[13] C. Scherer, P. Gahinet, and M. Ghilali. Multiobjective output-feedback control via LMI optimization. IEEE Transactions on Automatic Control, 42(7):896-911, 1997.

[14] T. Shimomura and T. Fujii. Multiobjective control design via successive overbounding of quadratic terms. In Proc. of the 39th CDC, pages 2763-2768, Sydney, Australia, 2000.

[15] O. Toker and H. Özbay. On the NP-hardness of solving bilinear matrix inequalities and simultaneous stabilization with static output feedback. In Proceedings of the American Control Conference, pages 2525-2526, Seattle, WA, 1995.

[16] H.D. Tuan and P. Apkarian. Low nonconvexity-rank bilinear matrix inequalities: Algorithms and applications in robust controller and structure designs. IEEE Transations on Automatic Control, 45(11):2111-2117, 2000.

[17] J.G. VanAntwerp and R.D. Braatz. A tutorial on linear and bilinear matrix inequalities. Journal of Process Control, 10:363-385, 2000.

[18] Y. Yamada and S. Hara. Global optimization for the $\mathcal{H}_{\infty}$ control with constant diagonal scaling. IEEE Transactions on Automatic Control, 43(2):191-203, 1998 